\title{
Pair production of $W$ bosons at the photon linear collider: a window to the electroweak symmetry breaking?
}

\author{
Georgy Jikia ${ }^{1}$ \\ Albert-Ludwigs-Universität Freiburg, Fakultät für Physik \\ Hermann-Herder Str.3, D-79104 Freiburg, Germany \\ and \\ Institute for High Energy Physics, Protvino \\ Moscow Region 142284, Russian Federation
}

\begin{abstract}
Recent progress in calculating $\mathcal{O}(\alpha)$ electroweak corrections to $W^{+} W^{-}$pair production in photon-photon collisions is reviewed. The potential of the Photon Linear Collider to study anomalous $W$ couplings is discussed.
\end{abstract}

\section{INTRODUCTION}

Linear colliders offer unique opportunities to study photon-photon collisions obtained using the process of Compton backscattering of laser light off electron beams from the linear collider [1]. This option is included now in conceptual design reports of the NLC, JLC and TESLA/SBLC projects $[3,4]$. The expected physics at the Photon Linear Collider (PLC) is very rich and complementary to that in $e^{+} e^{-}$collisions (see e.g. [2] and references therein).

Without the discovery of a light Higgs boson at LEP2, LHC or the linear collider, the best strategy to probe the symmetry breaking sector would lie in the study of the self couplings of the $W, Z$ bosons. The large cross sections for the processes involving $W$ 's make $\gamma \gamma$ colliders especially attractive tool in probing the $W$ self couplings. Indeed, the reaction $\gamma \gamma \rightarrow W^{+} W^{-}$would be the dominant source of the $W^{+} W^{-}$pairs at future linear colliders, provided that photon-photon collider option will be realized. The Born cross section of $W^{+} W^{-}$pair production in photon-photon collisions in the scattering angle interval $10^{\circ}<\theta^{ \pm}<170^{\circ}$ is $61 \mathrm{pb}$ at $\sqrt{s_{\gamma \gamma}}=500 \mathrm{GeV}$ and $37 \mathrm{pb}$ at $1 \mathrm{TeV}$. Corresponding cross sections of $W^{+} W^{-}$pair production in $e^{+} e^{-}$collisions are

1) Alexander von Humboldt Fellow; e-mail: jikia@phyv4.physik.uni-freiburg.de

(C) 1995 American Institute of Physics 
an order of magnitude smaller: $6.6 \mathrm{pb}$ at $500 \mathrm{GeV}$ and $2.5 \mathrm{pb}$ at $1 \mathrm{TeV}$. With more than a million $W W$ pairs per year a photon-photon collider can be really considered as a $W$-factory and an ideal place to conduct precision tests on the anomalous triple [5,8] and quartic [6-8] couplings of the $W$ bosons. In addition, in the process of triple $W W Z$ vector boson production it is possible to probe the tri-linear $Z W W$ and quartic couplings [7-10] as well as the $\mathcal{C}$ violating anomalous $Z W W, \gamma Z W W$ interactions [10].

With the natural order of magnitude on anomalous couplings one needs to know the $\mathcal{S M}$ cross sections with a precision better than $1 \%$ to extract these small numbers. From a theoretical point of view this calls for the very careful analysis of at least $\mathcal{O}(\alpha)$ corrections to the cross section of $W^{+} W^{-}$ pair production in $\gamma \gamma$ collisions, which were recently calculated including virtual corrections [11] and complete $\mathcal{O}(\alpha)$ corrections taking into account both virtual one-loop corrections and real photon and $Z$-boson emission [12].

\section{$\gamma \gamma \rightarrow W^{+} W^{-}$CROSS SECTIONS AND $\mathcal{O}(\alpha)$ CORRECTIONS}

Inclusive cross section of $W^{+} W^{-}$pair production in photon-photon collisions to third order in $\alpha$ is given by the IR-finite sum of Born cross section, interference term between the Born and one-loop amplitudes and cross section of $W^{+} W^{-}$pair production accompanied by the real photon emission. At energies above the $W W Z$ threshold one should add the cross section of $W^{+} W^{-} Z$ production.

$$
\begin{aligned}
& d \sigma\left(\gamma \gamma \rightarrow W^{+} W^{-}+X\right)= \\
& d \sigma^{\text {Born }}\left(\gamma \gamma \rightarrow W^{+} W^{-}\right)+\frac{1}{2 s_{\gamma \gamma}} 2 \operatorname{Re}\left(\mathcal{M}^{\text {Born }} \mathcal{M}^{1-\text { loop* }}\right) d P S^{(2)} \\
& +\left.d \sigma^{\text {soft }}\left(\gamma \gamma \rightarrow W^{+} W^{-} \gamma\right)\right|_{\omega_{\gamma}<k_{c}}+\left.d \sigma^{\text {hard }}\left(\gamma \gamma \rightarrow W^{+} W^{-} \gamma\right)\right|_{\omega_{\gamma}>k_{c}} \\
& +d \sigma^{Z}\left(\gamma \gamma \rightarrow W^{+} W^{-} Z\right) .
\end{aligned}
$$

In contrast to the corrections to the $Z$ boson production at LEP1, as a consequence of the universality of the electric charge no power or logarithmically enhanced corrections involving $m_{H}^{2} / M_{W}^{2}, \log \left(m_{H}^{2}\right), m_{t}^{2} / M_{W}^{2}, \log \left(m_{t}^{2}\right)$ or $\log \left(s / m_{f}^{2}\right)$ appear in the on-shell scheme in the limit, when Higgs-boson or top-quark masses are much larger than the collision energy or the fermion mass $m_{f}$ is much smaller than $\sqrt{s}[11,12]$.

Figure 1 shows total cross section of $W W$ pair production summed over $W W$ and $W W \gamma$ final states and integrated over $W^{ \pm}$scattering angles in the interval $10^{\circ}<\theta^{ \pm}<170^{\circ}$ as a function of energy for various polarizations. The bulk of the cross section originates from transverse $W_{T} W_{T}$ pair production. Transverse $W^{\prime}$ 's are produced predominantly in the forward/backward 

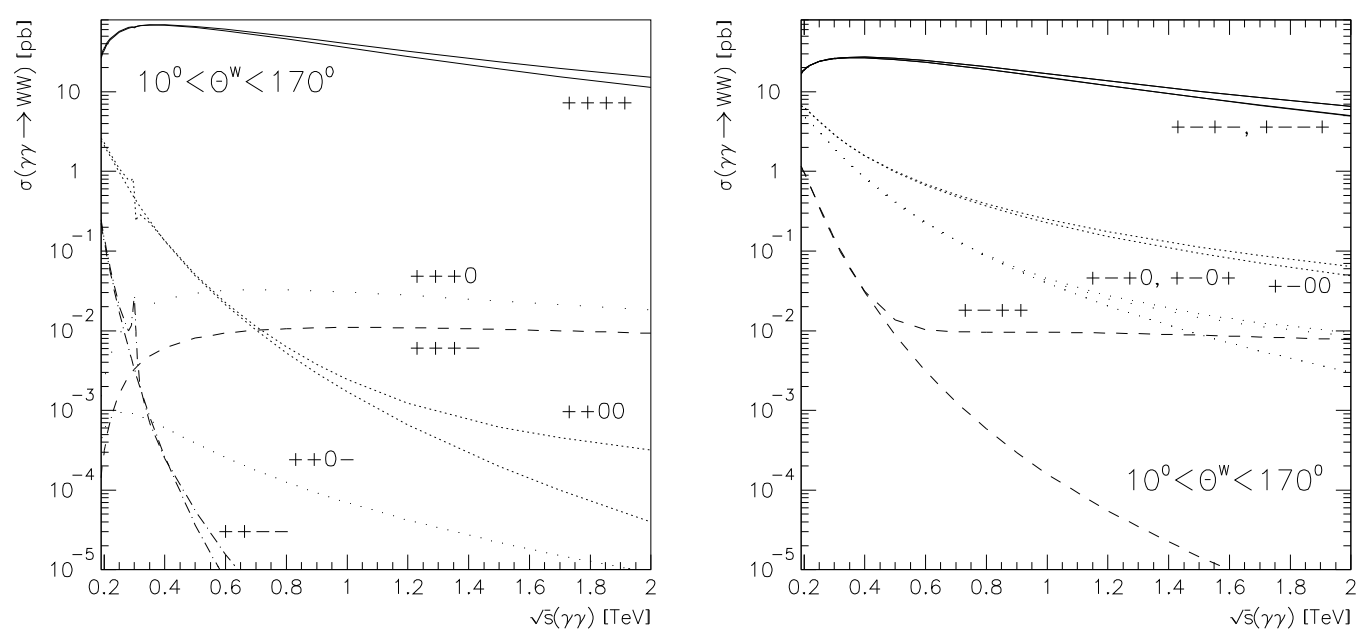

FIGURE 1. Total cross sections of $W W(\gamma)$ production for various polarizations. Born and corrected cross sections are shown. The curves nearest to the helicity notations represent the corrected cross sections.

direction and the helicity conserving amplitudes are dominating. Cross sections integrated over the whole phase space are non-decreasing with energy. For a finite angular cutoff they do decrease as $1 / s$, but still they are much larger than suppressed cross sections. For the dominating,+++++-+- , +--+ helicity configurations corrections are negative and they rise with energy ranging from $-3 \%$ at $500 \mathrm{GeV}$ to $-25 \%$ at $2 \mathrm{TeV}$. The correction for the next to the largest cross section $\sigma_{+-00}$ is also negative. For the other helicities radiative corrections are positive at high energies due to dominating positive contribution of real photon emission. Radiative corrections to cross sections $\sigma_{+-T L}$, which are decreasing at Born level as $1 / s^{2}$, are positive and large. For the cross sections $\sigma_{++00}$ and $\sigma_{+-++}$, which are decreasing as $1 / s^{3}$ for a finite angular cutoff, corrections are even larger. The cross section $\sigma_{++--}$is decreasing at tree level as $1 / s^{5}$ for a finite angular cutoff and is quite negligible at high energy. The cross sections $\sigma_{++L T}$ and $\sigma_{+++-}$vanish at the Born level, so only the process of $W W \gamma$ production contributes in Figure 1. The cross sections $\sigma_{++--}$and $\sigma_{++00}$ exhibit a clear clear Higgs resonance peak and the interference pattern, respectively, at $\sqrt{s_{\gamma \gamma}}=m_{H}=300 \mathrm{GeV}$.

In Table 1 Born cross sections and relative corrections are given for several intervals of $W^{ \pm}$scattering angles. At high energies large cancellations occur between negative virtual corrections and positive corrections corresponding to real photon or $Z$-boson emission. Consequently, although the correction originating from the $W W Z$ production is completely negligible at $\sqrt{s_{\gamma \gamma}}=$ $0.3 \mathrm{TeV}$, it is of the same order of magnitude as hard photon correction at $2 \mathrm{TeV}$. Although at $300 \div 500 \mathrm{GeV}$ corrections are quite small ranging from 
TABLE 1. Unpolarized Born cross sections and relative corrections for various intervals of $W^{ \pm}$scattering angles. Corrections originating from real hard photon $\left(\omega_{\gamma}>k_{c}=0.1 \mathrm{GeV}\right)$ and $Z$-boson emission as well as sum of soft photon and virtual boson contributions, fermion virtual corrections and total corrections are given separately.

\begin{tabular}{|c|c|c|c|c|c|c|}
\hline \multicolumn{7}{|c|}{$\sqrt{s}=300 \mathrm{GeV}$} \\
\hline$\theta_{W^{ \pm}},^{\circ}$ & $\sigma^{\text {Born }}, p b$ & $\delta^{\text {hard }}, \%$ & $\delta^{Z}, \%$ & $\delta^{\text {soft }+ \text { bose }}, \%$ & $\delta^{f e r m i}, \%$ & $\delta^{t o t}, \%$ \\
\hline$\overline{0^{\circ}<\theta<180^{\circ}}$ & 70.22 & 4.15 & $2.64 \cdot 10^{-2}$ & -7.09 & 0.327 & -1.37 \\
\hline $10^{\circ}<\theta<170^{\circ}$ & 64.46 & 4.11 & $2.74 \cdot 10^{-2}$ & -7.31 & 0.257 & -1.59 \\
\hline $30^{\circ}<\theta<150^{\circ}$ & 38.15 & 4.09 & $3.27 \cdot 10^{-2}$ & -8.62 & -0.123 & -2.67 \\
\hline $60^{\circ}<\theta<120^{\circ}$ & 12.96 & 4.02 & $2.94 \cdot 10^{-2}$ & -10.7 & -0.415 & -3.75 \\
\hline \multicolumn{7}{|c|}{$\sqrt{s}=500 \mathrm{GeV}$} \\
\hline$\theta_{W^{ \pm}}, \circ$ & $\sigma^{\text {Born }}, p b$ & $\delta^{\text {hard }}, \%$ & $\delta^{Z}, \%$ & $\delta^{\text {soft }+ \text { bose }}, \%$ & $\delta^{\text {fermi }}, \%$ & $\delta^{t o t}, \%$ \\
\hline$\overline{0^{\circ}<\theta<180^{\circ}}$ & 77.50 & 7.96 & 0.468 & -10.1 & $9.04 \cdot 10^{-2}$ & -1.63 \\
\hline $10^{\circ}<\theta<170^{\circ}$ & 60.71 & 7.89 & 0.541 & -10.7 & -0.242 & -2.52 \\
\hline $30^{\circ}<\theta<150^{\circ}$ & 21.85 & 8.05 & 0.817 & -13.0 & -1.34 & -5.50 \\
\hline $60^{\circ}<\theta<120^{\circ}$ & 5.681 & 8.02 & 0.789 & -14.8 & -2.13 & -8.12 \\
\hline \multicolumn{7}{|c|}{$\sqrt{s}=1000 \mathrm{GeV}$} \\
\hline$\theta_{W^{ \pm}},^{\circ}$ & $\sigma^{\text {Born }}, p b$ & $\delta^{\text {hard }}, \%$ & $\delta^{Z}, \%$ & $\delta^{\text {soft }+ \text { bose }}, \%$ & $\delta^{\text {fermi }}, \%$ & $\delta^{t o t}, \%$ \\
\hline$\overline{0^{\circ}<\theta<180^{\circ}}$ & 79.99 & 13.3 & 1.55 & -18.7 & $-5.51 \cdot 10^{-2}$ & -3.89 \\
\hline $10^{\circ}<\theta<170^{\circ}$ & 37.04 & 13.4 & 2.39 & -22.6 & -1.28 & -8.10 \\
\hline $30^{\circ}<\theta<150^{\circ}$ & 6.924 & 14.2 & 3.96 & -32.1 & -3.80 & -17.8 \\
\hline $60^{\circ}<\theta<120^{\circ}$ & 1.542 & 14.2 & 3.88 & -37.1 & -5.13 & -24.1 \\
\hline \multicolumn{7}{|c|}{$\sqrt{s}=2000 \mathrm{GeV}$} \\
\hline$\theta_{W^{ \pm}}{ }^{\circ}$ & $\sigma^{\text {Born }}, p b$ & $\delta^{\text {hard }}, \%$ & $\delta^{Z}, \%$ & $\delta^{\text {soft } t \text { bose }}, \%$ & $\delta^{\text {fermi }}, \%$ & $\delta^{t o t}, \%$ \\
\hline $0^{\circ}<\theta<180^{\circ}$ & 80.53 & 19.0 & 2.91 & -27.2 & $-7.45 \cdot 10^{-2}$ & -5.33 \\
\hline $10^{\circ}<\theta<170^{\circ}$ & 14.14 & 20.1 & 6.38 & -41.6 & -2.99 & -18.1 \\
\hline $30^{\circ}<\theta<150^{\circ}$ & 1.848 & 21.5 & 9.77 & -60.1 & -6.54 & -35.4 \\
\hline $60^{\circ}<\theta<120^{\circ}$ & 0.3936 & 21.6 & 9.60 & -67.6 & -8.04 & -44.5 \\
\hline
\end{tabular}

$-1.3 \%$ to $-8 \%$, depending on angular cuts, at $\mathrm{TeV}$ energies the value of radiative corrections in the central region of $W^{+} W^{-}$production become quite large, so that corrections in the region $60^{\circ}<\theta<120^{\circ}$ are $6 \div 8$ times larger than the corrections to the total cross section at $1 \div 2 \mathrm{TeV}$. They range from $-24 \%$ to $-45 \%$. Thus if precision measurements are to be made at TeV energy more careful theoretical analysis is needed in order to reliably predict the value of the cross section in the central region where the value of the cross section is the most sensitive to the $W$ anomalous couplings.

\section{DISCUSSION}

Although the cross section of $W W$ production is much larger in $\gamma \gamma$ collisions, this fact itself is not to be considered as an obvious advantage of PLC. The reason is that although the anomalous contribution to the amplitude of longitudinal $W_{L} W_{L}$ pair production is enhanced by a factor of $s / M_{W}^{2}$ both in $\gamma \gamma$ for $J_{z}=0$ (for $J_{z}= \pm 2$ in $\gamma \gamma$ collisions anomalous coupling contribu- 
tion is not enhanced at all) and $e^{+} e^{-}$collisions, the $\mathcal{S M}$ Born amplitude of $W_{L} W_{L}$ production at PLC is suppressed as $M_{W}^{2} / s$, so that the contribution of the interference term to the cross section is decreasing as $1 / s$ at PLC [13]. On the contrary, in $e^{+} e^{-}$collisions the anomalous contribution to the cross section is enhanced, corresponding to non-decreasing cross section of $W_{L} W_{L}$ production. Recently the authors of Ref. [13] have demonstrated that enhanced coupling could still be exploited in the $\gamma \gamma$ mode. Their clever idea is to reconstruct the non diagonal elements of the $W W$ polarization density matrix by analyzing the distributions of the decay products of the $W$ 's, thereby achieving the improvement over simple counting rate method of more that an order of magnitude at $\sqrt{s}=2 \mathrm{TeV}$. However, although the benefits from $\gamma \gamma$ mode are really quite evident at $\sqrt{s}=500 \mathrm{GeV}$ and allowed region in the anomalous couplings parameter space shrinks considerably when results from $e^{+} e^{-} \rightarrow W^{+} W^{-}$are combined with ones from $\gamma \gamma \rightarrow W^{+} W^{-}$, at energies above $1 \mathrm{TeV}$ combining results from $e^{+} e^{-}$and $\gamma \gamma$ modes does not considerably reduce the bounds obtained from $e^{+} e^{-} \rightarrow W^{+} W^{-}$alone [13]. This is especially true for fits with one anomalous coupling. Qualitatively these results can be understood considering the ratio $S / \sqrt{B}$ as a measure of statistical significance of the anomalous coupling signal $S$ with respect to the $\mathcal{S M}$ background $B$. Since the total $\mathcal{S M}$ cross section is decreasing as $1 / s$ in $e^{+} e^{-}$collisions and is constant in $\gamma \gamma$ collisions, while the enhanced anomalous cross section behaves like a constant we get

$$
\frac{S\left(e^{+} e^{-} \rightarrow W^{+} W^{-}\right)}{\sqrt{B\left(e^{+} e^{-} \rightarrow W^{+} W^{-}\right)}} \propto \sqrt{s}
$$

while $S\left(\gamma \gamma \rightarrow W^{+} W^{-}\right) / \sqrt{B\left(\gamma \gamma \rightarrow W^{+} W^{-}\right)} \propto 1$. If we take into account that anomalous couplings affect mostly the cross section in the central region, where the $\mathcal{S M}$ cross section behaves like $\sigma\left(\gamma \gamma \rightarrow W^{+} W^{-}\right) \sim 8 \pi \alpha^{2} / p_{T}^{2}$, we get

$$
\frac{S\left(\gamma \gamma \rightarrow W^{+} W^{-}\right)}{\sqrt{B\left(\gamma \gamma \rightarrow W^{+} W^{-}\right)}} \propto p_{T},
$$

i.e. the same improvement at higher energy as for $e^{+} e^{-}$collisions but only for large values of $p_{T}$ cut $p_{T} \sim s$, with which the cross section of $W W$ production in $\gamma \gamma$ collisions is not enhanced any more with respect to production in $e^{+} e^{-}$ collisions. Moreover, it is this region, where radiative corrections are as large as $-24 \div-45 \%$.

To resume, at $500 \mathrm{GeV}$ linear collider much stronger constraints on the anomalous $W$ couplings could be obtained combining the bounds from $e^{+} e^{-} \rightarrow$ $W^{+} W^{-}$and $\gamma \gamma \rightarrow W^{+} W^{-}$. At such an energy radiative corrections are well under control and photon-photon collider option is essential for precision measurements. 
At energies above $1 \mathrm{TeV}$, where enhancement factor $s / M_{W}^{2}$ for anomalous amplidudes starts playing a crucial role, PLC is not so much advantageous with respect to $e^{+} e^{-}$collider at the same integrated lumonosity. Radiative corrections are quite large and theoretical consideration beyond one-loop approximation is needed in order to safely predict $\mathcal{S M}$ cross section. Nevertheless, if in the photon-photon collisions one can obtain much larger luminosity than in $e^{+} e^{-}$collisions [1], the PLC potential could still be as high as at lower energies.

\section{ACKNOWLEDGMENTS}

I am grateful to the organizers of the Workshop for warm hospitality and to F. Boudjema, I. Ginzburg and V. Telnov for fruitful discussions. This work has been supported by the Alexander von Humboldt Foundation.

\section{REFERENCES}

1. V. Telnov, Proc. of the International Conference on the Structure and Interactions of the Photon, PHOTON '97, 10-15 May 1997, Egmond aan Zee, The Netherlands, physics/9706035.

2. G. Jikia, ibid., hep-ph/9706508.

3. NLC ZDR Design Group and NLC Physics Working Group (S. Kuhlman et al.), SLAC-R-0485, June 1996, hep-ex/9605011.

4. R. Brinkmann et al., DESY-79-048, July 1997, hep-ex/9707017.

5. S.Y.Choi, F.Shrempp, Phys.Lett. B272 (1991) 149; E.Yehudai, Phys. Rev. D44 (1991) 3434; A.Miyamoto in Proc. 2-nd Workshop on JLC, KEK 91-10 (1991).

6. G. Bélanger and F. Boudjema, Phys. Lett. B288 (1992) 210.

7. M. Baillargeon and F. Boudjema Phys. Lett. B317 (1993) 371.

8. M. Baillargeon, G. Belanger, and F. Boudjema, Proc. of the "Two-Photon Physics from DAФNE to LEP200 and Beyond", 2-4 February 1994, Paris.

9. F.T. Brandt, O.J.P. Éboli, E.M. Gregores, M.B. Magro, P.G. Mercadante and S.F. Novaes, Phys. Rev. D50 (1994) 5591.

10. M. Baillargeon, G. Bélanger, F. Boudjema and G. Jikia, Proceedings of the 3rd Workshop $e^{+} e^{-}$Collisions at TeV Energies: the Physics Potential, Part D, DESY, Hamburg, Germany, August 30 - September 1, 1995, p. 511.

11. A. Denner, S. Dittmaier, and R. Schuster, Nucl. Phys. B452 (1995) 80; Report BI-TP 96/03, WUE-ITP-96-001, hep-ph/9601355.

12. G. Jikia, Proc. of the Workshop Physics and Experiments with Linear $e^{+} e^{-}$ Colliders, Morioka, Japan, 1995; Nucl. Phys. B494 (1997) 19.

13. M. Baillargeon, G. Bélanger and F. Boudjema, preprint ENSLAPP-A-639/97, hep-ph/9701372. 